\title{
Dislocation Height Performs Well in Predicting the Use of Subtrochanteric Osteotomy in Crowe Type IV Hips
}

This article was published in the following Dove Press journal:

Therapeutics and Clinical Risk Management

\author{
Jingyang Sun ${ }^{1,2, *}$ \\ Guoqiang Zhang ${ }^{1,2, *}$ \\ Junmin Shen ${ }^{2,3}$ \\ Yinqiao Du (iD) ${ }^{2}$ \\ Bohan Zhang ${ }^{1,2}$ \\ Ming $\mathrm{Ni}^{1,2}$ \\ Yonggang Zhou (D) ${ }^{1,2}$ \\ Yan Wang ${ }^{1,2}$
}

'Medical School of Chinese PLA, Beijing I00853, People's Republic of China;

${ }^{2}$ Department of Orthopedics, The First Medical Center, Chinese PLA General Hospital, Beijing 100853, People's

Republic of China; ${ }^{3}$ Medical School of Nankai University, Tianjin 30007I,

People's Republic of China

*These authors contributed equally to this work
Correspondence: Yonggang Zhou; Yan Wang

Department of Orthopedics, The First Medical Center, Chinese PLA General Hospital, Fuxing Road, Haidian District, Beijing, People's Republic of China

Tel +86I380I287599; +86I380I379257

Email ygzhou30I@I63.com;

wangyyjspub@163.com
Purpose: The purpose of this study was to determine whether dislocation height can predict the use of subtrochanteric osteotomy in patients with Crowe type IV hip dysplasia.

Patients and Methods: We retrospectively included 102 patients affected by unilateral Crowe type IV developmental dysplasia who underwent primary total hip arthroplasty with modular cementless stem from April 2008 to May 2019 in our institution. Based on radiographs and operative notes, we found 62 hip arthroplasties were performed with subtrochanteric osteotomy and 40 without subtrochanteric osteotomy, which were named as the (subtrochanteric osteotomy) STO group and non-STO group, respectively. The predictive values of height of greater trochanter, height of femoral head/neck junction, and distalization of greater trochanter were analyzed using receiver operating characteristic (ROC) curves.

Results: The ROC curves showed that distalization of greater ntrochanter had the highest areas under the ROC curve (AUC), at 0.998. This was followed by height of greater trochanter and height of head/neck junction, which had AUCs of 0.937 and 0.935 , respectively. The optimal thresholds of these three indicators were $4.84 \mathrm{~cm}, 6.05 \mathrm{~cm}$, and $4.26 \mathrm{~cm}$. At the last follow-up, six dislocations occurred (five in the STO group and one in the nonSTO group). Four hips were treated by closed reduction and two by open reduction. Three patients (all in STO group) developed femoral nerve palsy with skin numbness on the frontal thigh or tibia and all recovered in a year. At outpatient visit, the limb length was measured. LLD was $<1 \mathrm{~cm}$ in $83 / 102,1-2 \mathrm{~cm}$ in $18 / 102$, and $>2 \mathrm{~cm}$ in $1 / 102$.

Conclusion: This study reveals that indicators of dislocation height are useful in predicting the use of subtrochanteric osteotomy during total hip arthroplasty for Crowe type IV hip dysplasia. However, a comprehensive, multivariate analysis may be required to validate these results.

Keywords: subtrochanteric osteotomy, developmental dysplasia of the hip, total hip arthroplasty, dislocation height

\section{Introduction}

Adult patients with developmental dysplasia of the hip (DDH) always develop secondary osteoarthritis and eventually end up with total hip arthroplasty (THA) at a younger age. ${ }^{1}$ Neglected or unsuccessfully treated Crowe type IV DDH is widely recognized as the most complicated case by arthroplasty surgeons. Altered anatomy of the acetabulum and proximal femur together with leg length discrepancy presents major challenges during THA in those patients. ${ }^{2}$ Due to biomechanical priority, the acetabular component is better to be implanted at the anatomical 
position, which may potentially require femoral shortening to avoid excessive limb lengthening and sciatic nerve injury. $^{2}$

Several methods have been described for femoral shortening in Crowe type IV hips, including greater trochanteric osteotomy with or without distal advancement, lesser trochanteric osteotomy, step-cut subtrochanteric osteotomy, oblique subtrochanteric osteotomy, V-shaped subtrochanteric osteotomy, transverse subtrochanteric osteotomy, and distal femoral osteotomy. ${ }^{3}$ Among these osteotomies, transverse subtrochanteric osteotomy was more widely used on the basis of reported literature. ${ }^{4,5}$ It has some advantages such as preserving of the proximal femoral metaphysis and simply adjusting the amount of bone resection.

However, some authors showed alternative surgical regimens of THA for Crowe type IV DDH to avoid femoral shortening, including intra-operative injection of muscle relaxant, more extensive soft tissue release, leverage and pre-operative iliofemoral distraction, and reported good clinical outcomes and acceptable complication rates. $^{6-9}$ It seems that not every dislocated hip necessitates femoral shortening when reducing the femoral head into true acetabulum during THA. In most studies, decisionmaking on femoral shortening before surgery was mainly based on radiographic templating. Femoral shortening was recommended when the lower limb would be excessively lengthened. The proposed threshold of limb lengthening varied greatly among different authors, mainly with the range from $3-5 \mathrm{~cm} .^{5,10}$ As the femoral canal in dysplastic hips is twisted, the minor axis of the isthmus appears on neither the anteroposterior nor the lateral radiograph. Thus measurements of isthmus size by radiographic templating will overestimate the minimum diameter of the canal. ${ }^{11}$

Since the anatomical abnormalities present are thought to increase with the severity of subluxation of the femur, the indicators which reflect the degree of subluxation may be useful for predicting the use of subtrochanteric osteotomy in total hip arthroplasty for Crowe type IV DDH. Therefore, the purpose of this study was to assess the association between dislocation height and subtrochanteric osteotomy.

\section{Patients and Methods}

\section{Patients}

After institutional review board approval, we retrospectively reviewed 171 consecutive patients affected by unilateral Crowe type IV DDH who underwent primary THA with modular cementless stem (S-ROM, DePuy, Warsaw, Indiana) from April 2008 to May 2019 in our institution. Exclusion criteria were 1) bilateral DDH, regardless of the subtype in contralateral hip, or 2) previous femoral osteotomy, or 3) previous hip pyogenic arthritis, or 4) proximal placement of the cup component, or 5) use of conical sleeve, or 6) inadequate post-operative anteroposterior radiographs. Finally, a total of 102 patients (102 hips) were included in the study (Figure 1).

Demographics and clinical information including age, gender, weight, height, and operative notes were collected from our electronic medical records. The application of subtrochanteric osteotomy was validated by osteotomy line on images of the second day after surgery and also by reference to operative notes.

\section{Radiographic Assessment}

Standardized digital, calibrated anteroposterior hip radiographs were acquired both pre-operatively and postoperatively. ${ }^{12}$ Radiographic assessment contained the measurement of dislocation height and distalization of greater trochanter (DGT). We adopted two methods to measure the dislocation height before surgery. One was the height of femoral head/neck junction (HJ) according to the Crowe classification. The other was the height of greater trochanter (HGT), which took the tip of greater trochanter as reference. The DGT was calculated by vertical height difference between the pre- and post-operative tip of the greater trochanter, which was perpendicular to the interteardrop line (Figure 2). Before embarking on the study, all observers reached an agreement on criteria for radiographic measurement. Removing all identifying marks, measurement was accomplished by two observers independently in random order, who were not involved in surgery. Then at least 1 month later, observers repeated their readings without knowledge of the previous results. All measurements were conducted by using Digimizer v5.4 (Acacialaan, Belgium). Inter-observer variability was measured by comparing the mean value of two observers on each occasion, while intra-observer reliability was determined by comparing the two reviews of each observer.

\section{Surgical Procedure}

All operations were performed by one senior surgeon under general anesthesia in the lateral decubitus position. The posterolateral approach was used in each case. Joint 


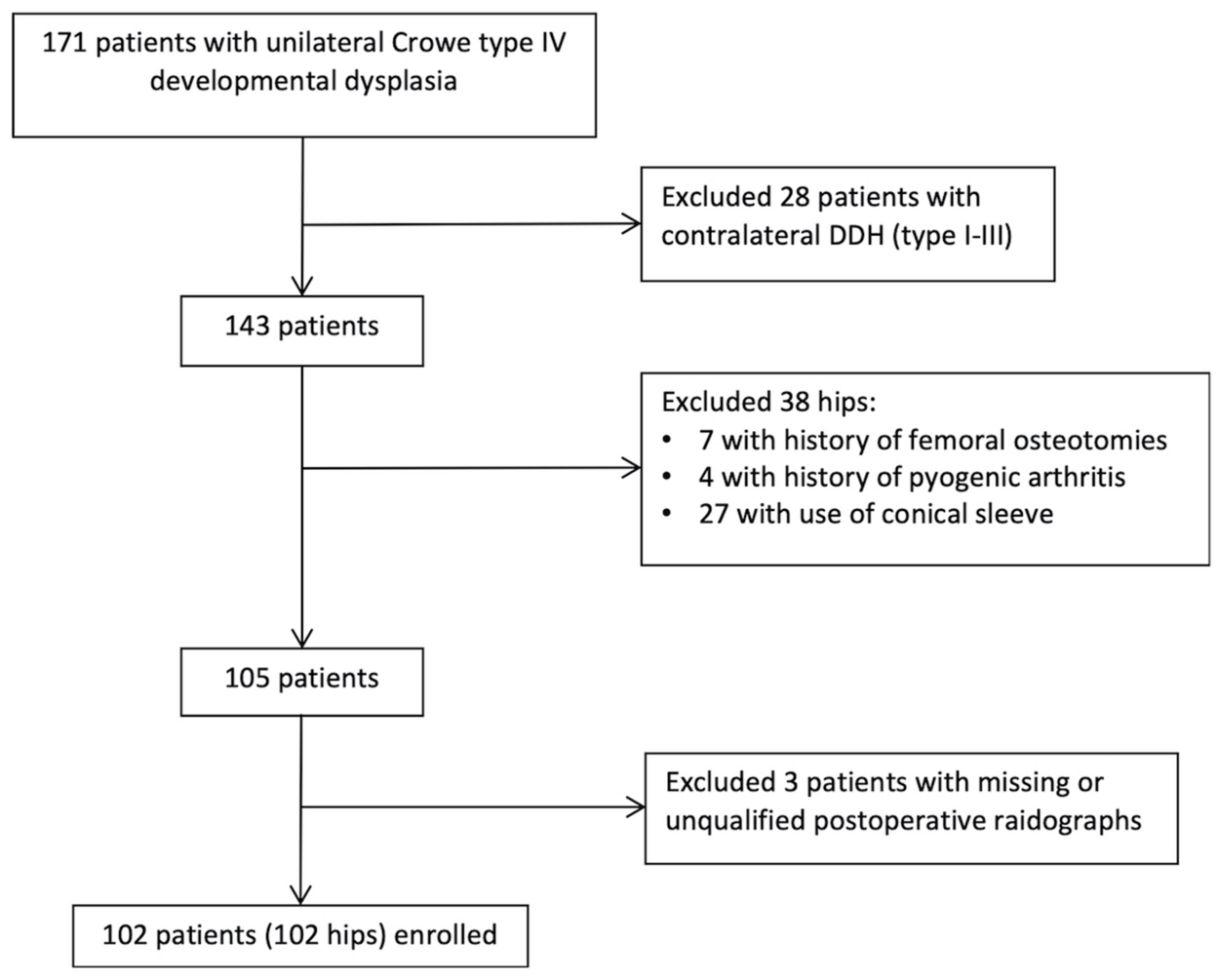

Figure I A flow diagram of the study.

capsulectomy, gluteal sling release, and iliopsoas tenotomy were conventionally performed. In order to use ceramic on ceramic bearing, the cup (range $=44-46 \mathrm{~mm}$ ) was implanted at the anatomic position by reaming the acetabulum posteriorly and inferiorly. ${ }^{13}$ Two or three screws were used to augment the primary stability of the cup. After the cup implantation, the femoral canal was prepared using the dedicated reamer for the S-ROM stem. First, we reamed the femoral canal until the maximum cortical contact was reached distally. Then conical and triangular reaming of the metaphysis was performed to prepare for the proximal sleeve. With trial seated in the femur, we measured the final vertical distance from the femoral head to the cup under constant and vigorous traction. If hip reduction with a femoral trial stem was impossible, a subtrochanteric osteotomy would be performed for femoral shortening. The osteotomy position was planned to be adjacent enough to the end of the proximal sleeve, approximately corresponding to $1-2 \mathrm{~cm}$ beneath the lesser trochanter. Prophylactic cerclage wires were placed both proximally and distally around the fragment of the planned osteotomy. Also, a longitudinal line along the femoral diaphysis was marked by electrocautery prior to osteotomy to determine the rotation. After removing the trial, a transverse osteotomy was performed, by resection of a length of the femur below the lesser trochanter. The length of the removed bone stock was based on the distance we measured before, leaving a scope of $1-1.5 \mathrm{~cm}$ with surgeon's discretion. After completion of osteotomy, a final preparation of the femur including repeated reaming and broaching was undertaken, until optimal cortical contact was achieved especially distal to the osteotomy site. Then trial reduction was performed again. If impossible, additional bone was incrementally resected at the osteotomy site until reduction was achieved. After trial reduction, stability, limb length, and soft tissue tension were evaluated. Intra-operative measurement of limb length discrepancy (LLD) was performed by palpating the inferior point of the bilateral patella. Mild LLD could be adjusted by means of the modifications of head/neck length and stem depth in the femur. Finally, the definitive femoral component was implanted with the rotational alignment of femoral stem adjusted to allow approximately $30-50^{\circ}$ of combined anteversion. During the stem insertion, compression of the osteotomy site was obtained without any gaps and no rotational adjustment of the bone segment was performed. At the end of the surgery, motion in abduction was assessed to evaluate the necessity of a percutaneous 


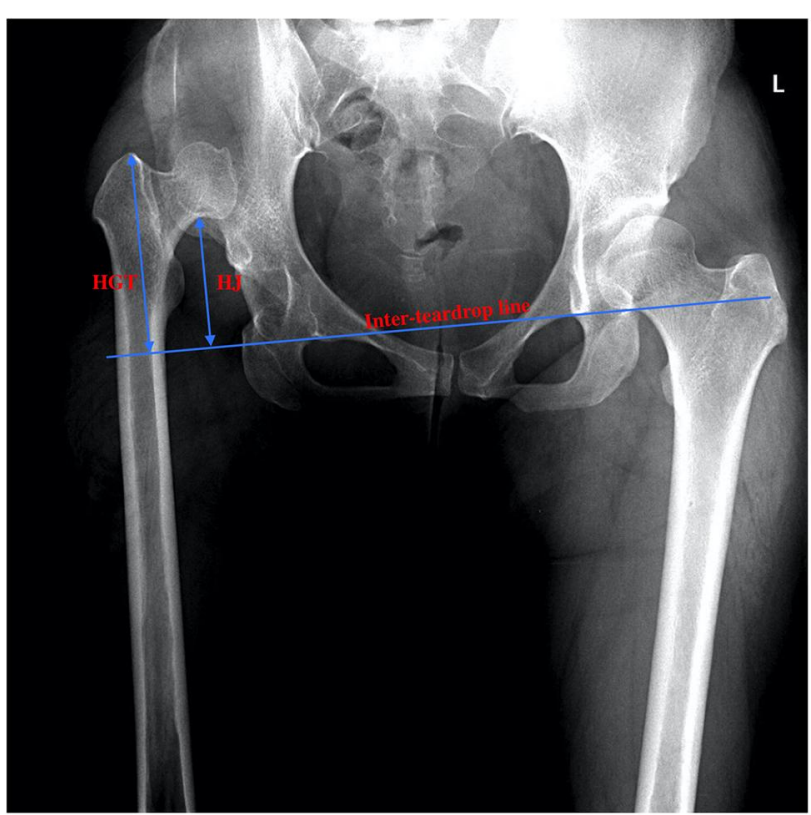

Figure 2 The measurement methods of dislocation height and distalization of greater trochanter. The dislocation height was presented as two indicators, which were perpendicular distances from the femoral head/neck junction and the tip of greater trochanter to the inter-teardrop line. The distalization of the greater trochanter was calculated by differences between the pre- and post-operative height of the greater trochanter.

Abbreviations: HGT, height of greater trochanter; HJ, height of femoral head/ neck junction.

partial adductor tenotomy. Post-operatively, patient's hip and knee were maintained in flexion for several days to relax the sciatic nerve and reduce tension of the soft tissue.

Patients were followed up in regular intervals at 3 months, 6 months, and yearly after surgery. Clinical information was collected, including LLD (limb length was measured from anterosuperior iliac spine to the prominence of medial malleolus), and occurrence of nerve injury and dislocation.

\section{Statistical Analysis}

Categorical variables were presented as frequencies, and continuous variables as means and standard deviation. Continuous variables were assessed using $t$-test, whereas categorical variables were analyzed using Chi-square test or Fisher's exact test. Assessment of inter- and intraobserver consistency was accomplished by the use of the intraclass correlation coefficient (ICC). Agreement was graded as slight (ICC $=0-0.2$ ), fair $(\mathrm{ICC}=0.21-0.40)$, moderate ( $\mathrm{ICC}=0.41-0.60)$, substantial $(\mathrm{ICC}=0.61-0.80$ ), or almost perfect $(\mathrm{ICC}=0.81-1.0)$. Receiver operating characteristic (ROC) curves were generated to determine the predicting value of each indicator for the assessment of subtrochanteric osteotomy. The area under the curve (AUC) and 95\% confidence interval (CI) were calculated. The discriminatory value of curves was interpreted as excellent (0.9-1), good (0.8-0.89), fair (0.7-0.79), poor (0.6-0.69), or as failing or having no discriminatory capacity $(0.5-0.59)$. The optimal threshold for an indicator as a predicting tool for subtrochanteric osteotomy was determined using the Youden index. The sensitivity, specificity, positive predictive value (PPV), and negative predictive value (NPV) of the indicators were calculated. All statistical analyses were performed using SPSS version 26.0 (IBM Inc., Armonk, NY). $P$-value of $<0.05$ was considered significant.

\section{Results}

According to the review of radiographs and operative notes, 62 hips with subtrochanteric osteotomy and 40 hips without subtrochanteric osteotomy were included in the study, which were named the STO group and non-STO group, respectively (Figures 3 and 4). There were no significant differences in demographic data between the two groups $(P>0.05$, Table 1$)$.

The intra-observer and inter-observer agreements were found to have almost perfect reliability for all of the measurements (ICC $>0.81)$. The results are shown in Table 2.

HGT was $7.77 \mathrm{~cm}$ (range $=4.96-10.60 \mathrm{~cm}$ ) in the STO group and $5.08 \mathrm{~cm}($ range $=2.89-7.26 \mathrm{~cm})$ in the non-STO group $(P<0.001)$. HJ was $5.46 \mathrm{~cm}$ (range $=3.26-8.10 \mathrm{~cm})$ in the STO group and $3.45 \mathrm{~cm}$ (range $=1.86-5.47 \mathrm{~cm}$ ) in the non-STO group $(P<0.001)$. DGT was also evaluated, with the results of $6.68 \mathrm{~cm}$ (range $=4.33-8.78 \mathrm{~cm}$ ) and $3.04 \mathrm{~cm}$ (range $=1.56-4.81 \mathrm{~cm})(P<0.001)$.

The ROC curves showed that DGT had the highest AUC, at 0.998 . This was followed by HGT and HJ, which had AUCs of 0.937 and 0.935 , respectively. The AUCs of these three indicators were all beyond 0.9, indicating that they had excellent performance in predicting the use of subtrochanteric osteotomy (Figure 5). Based on our results of ROC curve analysis, the optimal threshold, sensitivity, specificity, positive predictive value (PPV), and negative predictive value (NPV) are summarized in Table 3.

At the last follow-up, six dislocations (at 1 month, 2 months, and 3 months) occurred (five in the STO group and one in the non-STO group). Four hips were treated by closed reduction and two by open reduction. Three patients (all in the STO group) developed femoral 


\section{A}

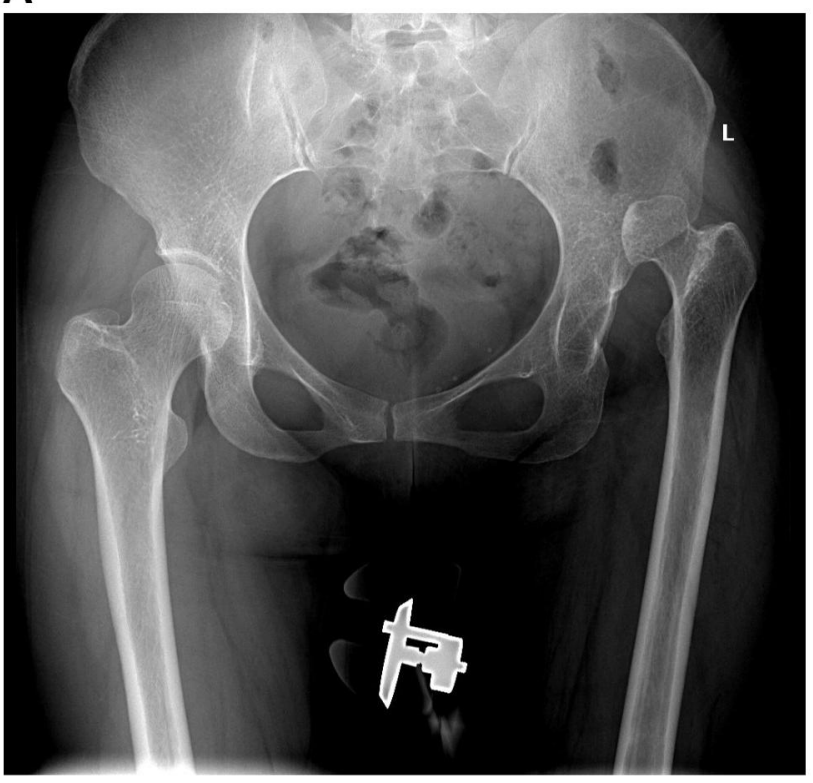

B

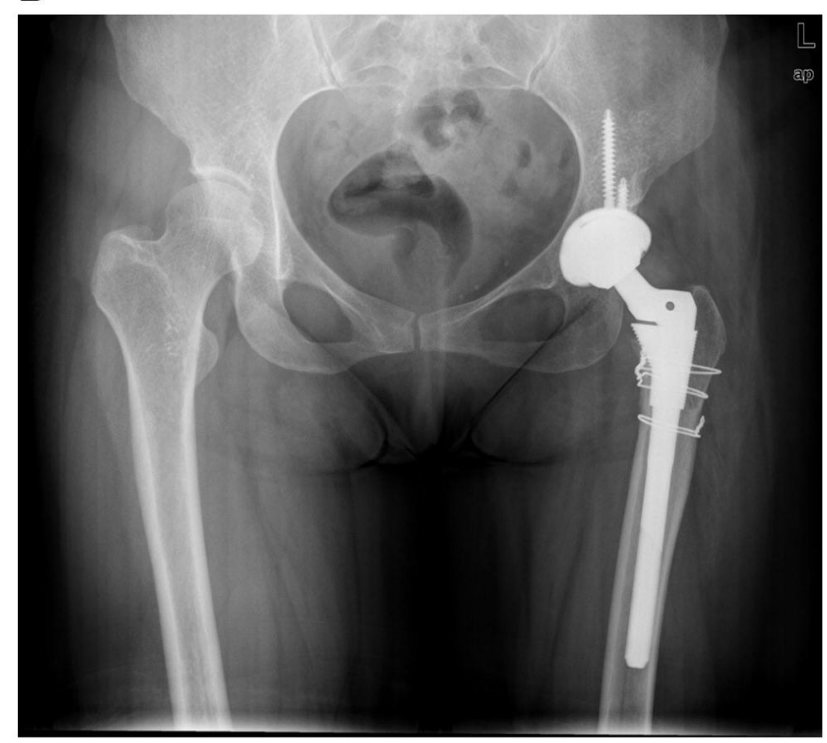

Figure 3 X-ray of a 42-year-old woman undergoing THA with subtrochanteric osteotomy. (A) Pre-operative X-ray. (B) Post-operative X-ray.

nerve palsy with skin numbness on the frontal thigh or tibia and all recovered in a year. At outpatient visit, the limb length was measured. LLD was $<1 \mathrm{~cm}$ in $83 / 102$ (48 in the STO-group and 35 in the non-STO group), $1-2 \mathrm{~cm}$ in $18 / 102$ (13 in the STO group and five in the non-STO group), and $>2 \mathrm{~cm}$ in $1 / 102$ (in the $\mathrm{STO}$ group) $(P=0.380)$.

\section{Discussion}

According to Crowe classification, anatomical abnormalities are often thought to increase with higher femoral subluxation. $^{2}$ Subtrochanteric osteotomy is likely to be
A

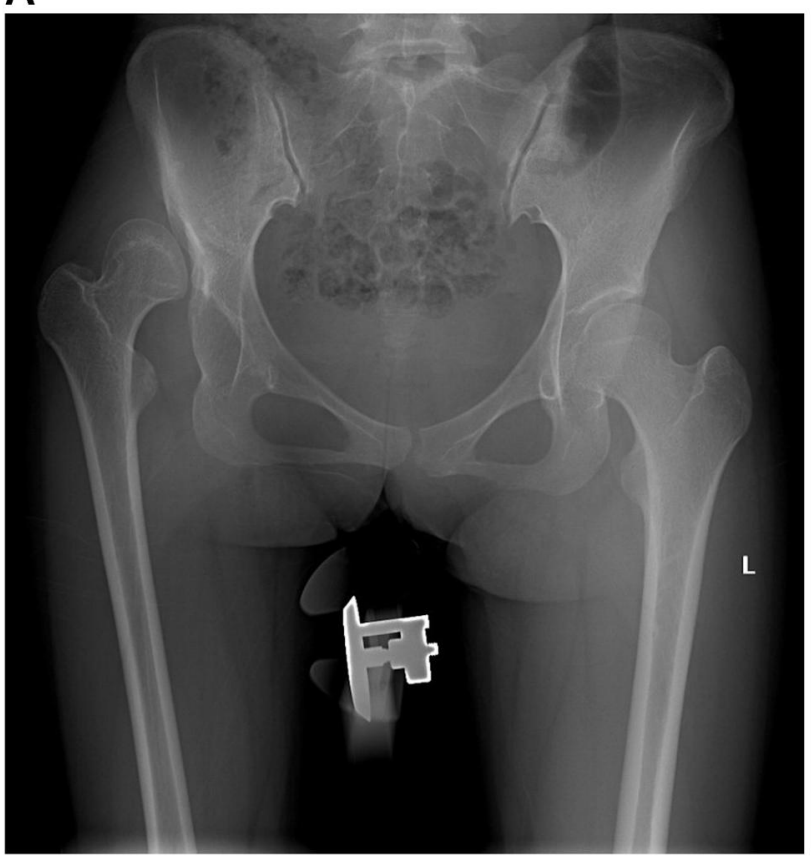

B

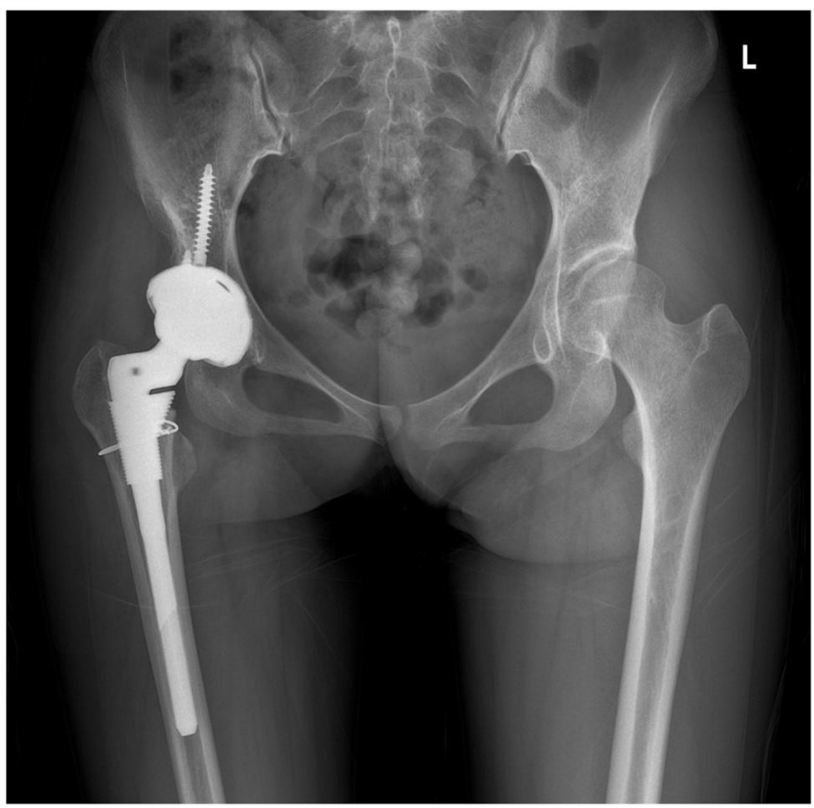

Figure $4 X$-ray of a 24-year-old woman undergoing THA without subtrochanteric osteotomy. (A) Pre-operative X-ray. (B) Post-operative X-ray.

performed in irreducible Crowe type IV hips during THA. However, current studies reported similarly good outcomes of THA with or without femoral shortening in these patients. ${ }^{6,8,14-16}$ We supposed that all Crowe type IV hips were not the same. And subtrochanteric osteotomy might be especially appropriate for some cases. To our best knowledge, this is the first study to evaluate the dislocation height for predicting the use of subtrochanteric 
Table I Patient Demographics of the STO and Non-STO Groups

\begin{tabular}{|l|l|l|l|}
\hline & $\begin{array}{l}\text { STO Group } \\
(\mathbf{n = 6 2 )}\end{array}$ & $\begin{array}{l}\text { Non-STO Group } \\
\mathbf{( n = 4 0 )}\end{array}$ & P-value \\
\hline $\begin{array}{l}\text { Male/female } \\
\text { sex, } \mathrm{n}\end{array}$ & $7 / 55$ & $2 / 38$ & 0.274 \\
Age (year) & $41.62 \pm 13.34$ & $37.74 \pm 9.49$ & 0.100 \\
Height (m) & $1.57 \pm 0.06$ & $1.57 \pm 0.08$ & 0.967 \\
Weight $(\mathrm{kg})$ & $56.78 \pm 10.30$ & $55.95 \pm 6.97$ & 0.636 \\
BMI $\left(\mathrm{kg} / \mathrm{m}^{2}\right)$ & $22.98 \pm 3.62$ & $22.82 \pm 2.83$ & 0.837 \\
\hline
\end{tabular}

Abbreviations: STO, subtrochanteric osteotomy; BMI, body mass index.

osteotomy. The results revealed that both HGT and HJ were potent indicators, with AUCs of 0.937 and 0.935, respectively.

Previous studies have recommended varying thresholds of limb lengthening, mainly with the range from $3-5 \mathrm{~cm} .{ }^{5,10}$ In this study, we also measured vertical height difference between the pre- and post-operative tip of the greater trochanter, which actually indicated the migration of proximal femur during reduction of the hip. As an indicator, DGT performed very well in predicting the use of subtrochanteric osteotomy and had an excellent AUC (0.998). Its optimal threshold was calculated to be $4.84 \mathrm{~cm}$, which was close to the value $(5 \mathrm{~cm})$ proposed by Higuchi et al. ${ }^{10}$ However, DGT was more difficult to acquire than HGT and HJ before surgery. Due to the torsional femoral canal in high dislocated hips, templating with plain radiographs may wrongly define the stem size and its insertion depth, which makes the measurement of DGT inaccurate. ${ }^{11}$ Perhaps DGT will exert its function in a three-dimensional pre-operative planning. ${ }^{17}$

On the basis of our data, both HGT and HJ almost performed as well as DGT, which were beneficial for predicting the use of subtrochanteric osteotomy before surgery. The former two indicators might contribute more to clinical practice due to their accessibility on plain radiographs. Before initiation of this study, we thought HJ would perform more poorly than HGT. On the one hand, the position of head/neck junction can be changeful because of the varying neck-shaft angles among Crowe type IV hips

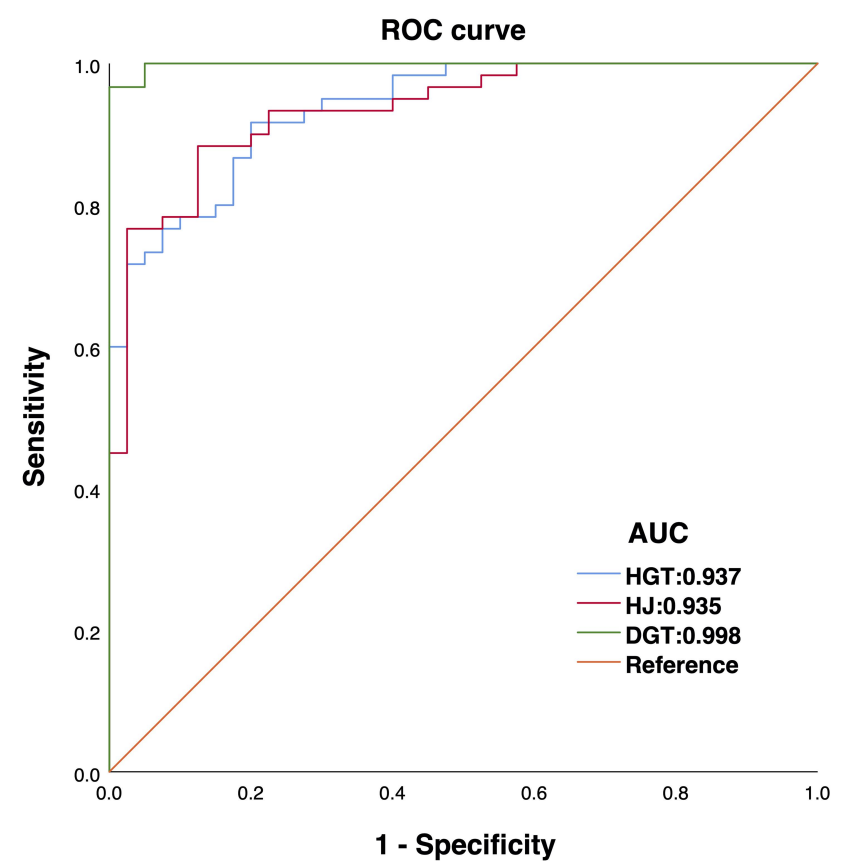

Figure 5 ROC curves for dislocation height and distalization of greater trochanter in predicting the use of subtrochanteric osteotomy.

Abbreviations: DGT, distalization of greater trochanter; HGT, height of greater trochanter; $\mathrm{HJ}$, height of femoral head/neck junction.

(Figure 6A-D). On the other, greater trochanter, as the insertion of abductor muscle, the height of which may be more associated with the contracture and shortening course of musculature. Unexpectedly, they did equally well regarding the predictive value. However, considering the morphological complexity of femoral head, $\mathrm{HJ}$ is often sometimes hard to measure, especially in the cases with epiphyseal slippage, severe iliofemoral osteoarthritis, or even an absorbed femoral head (Figure 6E-H). Though a more stable landmark, the size of the great trochanter may vary from person to person, which partly explains the relatively lower specificity of HGT.

What's more, we have to admit that there are several other factors which may influence the decision of subtrochanteric osteotomy, such as proximal femoral deformity, hardware retention, and medullary occlusion secondary to pyogenic arthritis. In those cases, subtrochanteric osteotomy may be required regardless of the dislocation height. ${ }^{18,19}$

Table 2 Reliability of Length Measurements

\begin{tabular}{|l|l|l|l|}
\hline Observer & HGT, 95\% Cl & HJ, 95\% Cl & DGT, 95\% Cl \\
\hline $\mathbf{I}$ & $0.950(0.926-0.966)$ & $0.951(0.928-0.967)$ & $0.955(0.934-0.969)$ \\
2 & $0.942(0.915-0.960)$ & $0.946(0.921-0.963)$ & $0.995(0.993-0.997)$ \\
Interobserver & $0.955(0.935-0.970)$ & $0.913(0.873-0.940)$ & $0.979(0.969-0.986)$ \\
\hline
\end{tabular}


Table 3 The Predictive Values for Three Indicators

\begin{tabular}{|l|l|l|l|l|l|l|}
\hline Indicators & AUC $(\mathbf{9 5} \% \mathbf{C l})$ & Optimal Threshold & Sensitivity & Specificity & PPV & NPV \\
\hline HGT & $0.937(0.894-0.980)$ & 6.05 & 0.917 & 0.800 & 0.877 & 0.865 \\
HJ & $0.935(0.889-0.981)$ & 4.26 & 0.883 & 0.875 & 0.914 & 0.833 \\
DGT & $0.998(0.995-1.000)$ & 4.84 & 0.967 & 1.000 & 1.000 & 0.952 \\
\hline
\end{tabular}

Abbreviations: HGT, height of greater trochanter; HJ, height of femoral head/neck junction; DGT, distalization of greater trochanter.

In this study, only modular stems with triangular sleeves were used. Although data appeared more homogeneous, results of one single femoral prosthesis would limit general applicability of the optimal thresholds we determined. However, S-ROM stem has been widely used and good outcomes have been reported in vast literatures. ${ }^{5,20}$ It has advantages in adjusting the stem anteversion within a torsional femur, addressing the mismatch of the metaphysis and diaphysis, and enhancing the rotational stability when subtrochanteric osteotomy is performed. Another commonly used implant which can address both excessive anteversion and stability of the osteotomy site is the conical tapered stem with splines (Wagner Cone, Zimmer, Warsaw, IN, USA) ${ }^{4,21}$ Due to its overall conical shape, the Wagner Cone stem may be easier to be inserted deeply, leaving a smaller portion of the stem suspending on the proximal femur. Namely, compared with the S-ROM stem with triangular sleeve, a conical stem may accommodate higher dislocation with no aid of femoral shortening. That is why we remove the cases with use of conical sleeve, which is a special type of proximal component in S-ROM prosthesis.

There are several limitations in this study. First, this was a retrospective study. Fortunately, we mainly relied on the radiographs with less effect from recall bias. Second, several confounding factors which may influence the decision of subtrochanteric osteotomy have been ruled out. Therefore, a comprehensive, multivariate analysis may be needed in the future. Additionally, this study was designed as a diagnostic test and not much attention has been given to the outcome of THA with or without subtrochanteric osteotomy. Finally, it was a single-institution, singlesurgeon study with limited external validity. However, with the excellent predictive accuracy, dislocation height can indeed remind arthroplasty surgeons of the need for subtrochanteric osteotomy.

\section{Conclusion}

This study reveals that HGT and HJ are potent indicators in predicting the use of subtrochanteric osteotomy during
A

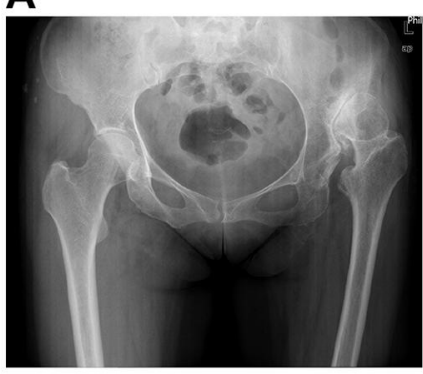

E

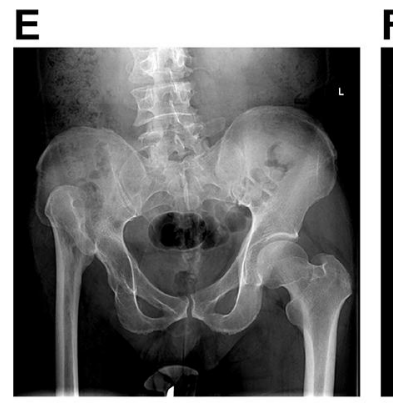

B

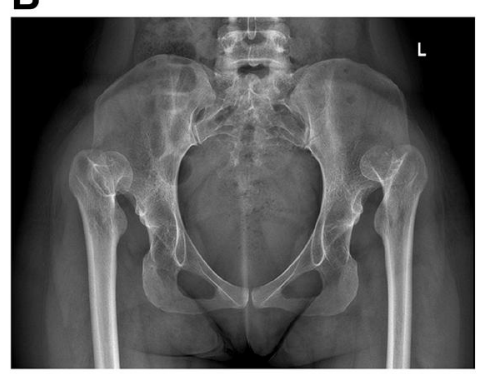

F

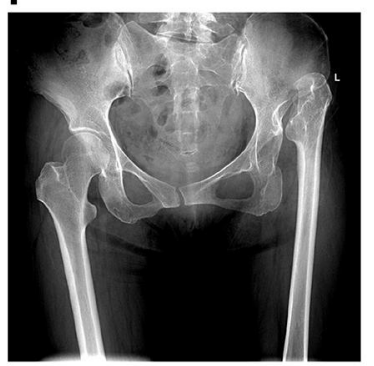

C

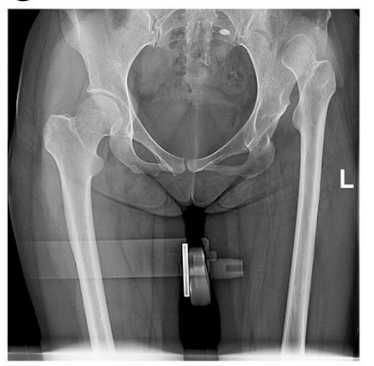

H

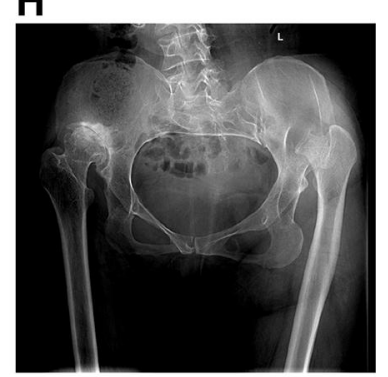

Figure 6 (A-D) The position of head/neck junction can be changeful because of the varying neck-shaft angles. (E-H) Due to the morphological complexity of femoral head, the head/neck junction is sometimes hard to measure, especially in the cases with severe abrasion or absence of the femoral head. 
THA for Crowe type IV DDH, with optimal thresholds of $6.05 \mathrm{~cm}$ and $4.26 \mathrm{~cm}$, respectively. However, due to the common morphological complexity and previous surgical histories in these patients, a comprehensive, multivariate analysis may be required to validate these results.

\section{Ethical Approval}

This retrospective review study involving human participants was in accordance with the ethical standards of the institutional and national research committee and with the 1964 Helsinki Declaration and its later amendments or comparable ethical standards. This study was approved by the medical ethics committee of Chinese PLA General Hospital (S2020-138-01).

\section{Informed Consent}

Informed consent was obtained from all individual participants included in the study.

\section{Acknowledgments}

The authors would like to thank all staff from the participating departments and clinics.

\section{Funding}

This research did not receive any specific grant from funding agencies in the public, commercial, or not-forprofit sectors.

\section{Disclosure}

The authors report no conflicts of interest in this work. The first two authors contributed equally to this work and are considered co-first authors.

\section{References}

1. Kosuge D, Yamada N, Azegami S, et al. Management of developmental dysplasia of the hip in young adults: current concepts. Bone Joint J. 2013;95-B(6):732-737. doi:10.1302/0301-620x.95b6.31286

2. Greber EM, Pelt CE, Gililland JM, et al. Challenges in total hip arthroplasty in the setting of developmental dysplasia of the hip. J Arthroplasty. 2017;32(9):S38-S44. doi:10.1016/j.arth.2017.02.024

3. Yang S, Cui Q. Total hip arthroplasty in developmental dysplasia of the hip: review of anatomy, techniques and outcomes. World J Orthop. 2012;3(5):42-48. doi:10.5312/wjo.v3.i5.42

4. Grappiolo G, La Camera F, Della Rocca A, et al. Total hip arthroplasty with a monoblock conical stem and subtrochanteric transverse shortening osteotomy in crowe type IV dysplastic hips. Int Orthop. 2019;43 (1):77-83. doi:10.1007/s00264-018-4122-5

5. Wang D, Li LL, Wang HY, et al. Long-term results of cementless total hip arthroplasty with subtrochanteric shortening osteotomy in crowe type IV developmental dysplasia. $J$ Arthroplasty. 2017;32 (4):1211-1219. doi:10.1016/j.arth.2016.11.005
6. Yan F, Chen G, Yang L, et al. A reduction technique of arthroplasty without subtrochanteric femoral shortening osteotomy for the treatment of developmental high dislocation of hip: a case series of 28 hips. J Arthroplasty. 2014;29(12):2289-2293. doi:10.1016/j.arth. 2013.11.016

7. Wu X, Li SH, Lou LM, et al. The techniques of soft tissue release and true socket reconstruction in total hip arthroplasty for patients with severe developmental dysplasia of the hip. Int Orthop. 2012;36 (9):1795-1801. doi:10.1007/s00264-012-1622-6

8. Li H, Yuan Y, Xu J, et al. Direct leverage for reducing the femoral head in total hip arthroplasty without femoral shortening osteotomy for crowe type 3 to 4 dysplasia of the hip. J Arthroplasty. 2018;33 (3):794-799. doi:10.1016/j.arth.2017.09.011

9. Binazzi R. Two-stage progressive femoral lowering followed by cementless total hip arthroplasty for treating crowe IV-hartofilakidis type 3 developmental dysplasia of the hip. J Arthroplasty. 2015;30 (5):790-796. doi:10.1016/j.arth.2014.12.019

10. Higuchi $Y$, Hasegawa Y, Ishiguro N. Leg lengthening of more than $5 \mathrm{~cm}$ is a risk factor for sciatic nerve injury after total hip arthroplasty for adult hip dislocation. Nagoya J Med Sci. 2015;77(3):455-463.

11. Argenson JN, Ryembault E, Flecher X, et al. Three-dimensional anatomy of the hip in osteoarthritis after developmental dysplasia. J Bone Joint Surg Br. 2005;87-B(9):1192-1196. doi:10.1302/0301620x.87b9.15928

12. Cooke TD, Scudamore RA, Bryant JT, et al. A quantitative approach to radiography of the lower limb. Principles and applications. J Bone Joint Surg Br. 1991;73-B(5):715-720. doi:10.1302/0301620X.73B5.1894656

13. Zhou Y, Sun C, Wang Y. New method addressing the problem of using ceramic-on-ceramic bearing in too small acetabulum of high-riding DDH patients with THA. Semin Arthroplasty. 2012;23 (4):226-231. doi:10.1053/j.sart.2012.12.006

14. Chu YM, Zhou YX, Han N, et al. Two different total hip arthroplasties for hartofilakidis type $\mathrm{C} 1$ developmental dysplasia of hip in adults. Chin Med J. 2016;129(3):289-294. doi:10.4103/03666999.174507

15. Li H, Xu J, Qu X, et al. Comparison of total hip arthroplasty with and without femoral shortening osteotomy for unilateral mild to moderate high hip dislocation. $J$ Arthroplasty. 2017;32(3):849-856. doi:10.1016/j.arth.2016.08.021

16. Necas L, Hrubina M, Melisik M, et al. Cementless hip arthroplasty and transverse shortening femoral osteotomy with the S-ROM stem for crowe type IV developmental dysplasia. Eur J Orthop Surg Traumatol. 2019;29(5):1025-1033. doi:10.1007/s00590-019-02400-y

17. Sariali E, Catonne Y, Pascal-Moussellard H. Three-dimensional planning-guided total hip arthroplasty through a minimally invasive direct anterior approach. Clinical outcomes at five years' follow-up. Int Orthop. 2017;41(4):699-705. doi:10.1007/s00264-016-3242-z

18. Perry KI, Berry DJ. Femoral considerations for total hip replacement in hip dysplasia. Orthop Clin North Am. 2012;43(3):377-386. doi:10.1016/j.ocl.2012.05.010

19. Rosenstein AD, Diaz RJ. Challenges and solutions for total hip arthroplasty in treatment of patients with symptomatic sequelae of developmental dysplasia of the hip. Am J Orthop. 2011;40(2):87-91.

20. Wang S, Zhou Y, Ma H, et al. Mid-term results of total hip replacement with subtrochanteric osteotomy, modular stem, and ceramic surface in crowe IV hip dysplasia. Arthroplast Today. 2018;4 (3):363-369. doi:10.1016/j.artd.2017.07.003

21. Tahta M, Isik C, Uluyardimci E, et al. Total hip arthroplasty without subtrochanteric femoral osteotomy is possible in patients with crowe III/IV developmental dysplasia: total hip arthroplasty without femoral osteotomy. Arch Orthop Trauma Surg. 2020;140(3):409-413. doi:10.1007/s00402-019-03320-6 


\section{Publish your work in this journal}

Therapeutics and Clinical Risk Management is an international, peerreviewed journal of clinical therapeutics and risk management, focusing on concise rapid reporting of clinical studies in all therapeutic areas, outcomes, safety, and programs for the effective, safe, and sustained use of medicines. This journal is indexed on PubMed Central, CAS,
EMBase, Scopus and the Elsevier Bibliographic databases. The manuscript management system is completely online and includes a very quick and fair peer-review system, which is all easy to use. Visit http://www.dovepress.com/testimonials.php to read real quotes from published authors.

Submit your manuscript here: https://www.dovepress.com/therapeutics-and-clinical-risk-management-journal 